\title{
The Preparation and Characterization of Chitosan-Based Hydrogels Cross-Linked by Glyoxal
}

\author{
Beata Kaczmarek-Szczepańska ${ }^{1, *(1)}$, Olha Mazur ${ }^{1}$, Marta Michalska-Sionkowska ${ }^{2}{ }^{\circledR}$, Krzysztof Łukowicz ${ }^{3}$ \\ and Anna Maria Osyczka ${ }^{3}$ \\ 1 Department of Biomaterials and Cosmetics Chemistry, Faculty of Chemistry, Nicolaus Copernicus University \\ in Toruń, Gagarin 7, 87-100 Toruń, Poland; 289185@stud.umk.pl \\ 2 Department of Environmental Microbiology and Biotechnology, Faculty of Biology and Veterinary Science, \\ Nicolaus Copernicus University in Toruń, Lwowska 1, 87-100 Toruń, Poland; mms@umk.pl \\ 3 Department of Cell Biology and Imaging, Institute of Zoology and Biomedical Research, Faculty of Biology, \\ Jagiellonian University, Gronostajowa 7, 30-387 Kraków, Poland; krzysztof.lukowicz@uj.edu.pl (K.Ł.); \\ anna.osyczka@uj.edu.pl (A.M.O.) \\ * Correspondence: beata.kaczmarek@umk.pl
}

check for updates

Citation: Kaczmarek-Szczepańska, B.; Mazur, O.; Michalska-Sionkowska, M.; Łukowicz, K.; Osyczka, A.M. The Preparation and Characterization of Chitosan-Based Hydrogels Cross-Linked by Glyoxal. Materials 2021, 14, 2449. https://doi.org/ $10.3390 /$ ma14092449

Academic Editor:

Assunta Borzacchiello

Received: 1 April 2021

Accepted: 5 May 2021

Published: 9 May 2021

Publisher's Note: MDPI stays neutral with regard to jurisdictional claims in published maps and institutional affiliations.

Copyright: (c) 2021 by the authors. Licensee MDPI, Basel, Switzerland. This article is an open access article distributed under the terms and conditions of the Creative Commons Attribution (CC BY) license (https:/ / creativecommons.org/licenses/by/ $4.0 /)$.

\begin{abstract}
In this study, hydrogels based on chitosan cross-linked by glyoxal have been investigated for potential medical applications. Hydrogels were loaded with tannic acid at different concentrations. The thermal stability and the polyphenol-releasing rate were determined. For a preliminary assessment of the clinical usefulness of the hydrogels, they were examined for blood compatibility and in the culture of human dental pulp cells (hDPC). The results showed that after immersion in a polyphenol solution, chitosan/glyoxal hydrogels remain nonhemolytic for erythrocytes, and we also did not observe the cytotoxic effect of hydrogels immersed in tannic acid (TA) solutions with different concentration. Tannic acid was successfully released from hydrogels, and its addition improved material thermal stability. Thus, the current findings open the possibility to consider such hydrogels in clinics.
\end{abstract}

Keywords: hydrogels; chitosan; glyoxal; tannic acid; cells study

\section{Introduction}

Currently, designing active substance delivery systems constitute one of the goals of novel materials development. The release of active compounds directly to the infected site provides the highest effectiveness of treatment [1]. Chitosan is one of the best-known polysaccharides that may be used to obtain biocompatible materials [2]. It may be isolated from food industry by-products as shells of crustaceans, which provides chitosan to be easily accessible. Essentially, material preparation methods that are cheap and fast should be considered since only those may find industrial applications.

Hydrogels based on chitosan have to be cross-linked to present improved material stability, as chitosan dissolves in aqueous-like conditions very easily. However, the solubility of chitosan depends on the molecular weight and deacetylation degree of chitosan as well as the $\mathrm{pH}$ of the solution. Various compounds are known to be applicable as cross-linker agents [3].

Materials may be enriched with active substances, for instance polyphenols. Tannic acid is the example of a polyphenol that shows numerous important properties. To our knowledge, the proposed tannic acid-loaded hydrogels preparation method is a novelty. Tannic acid provides antimutagenic, antitumor, and antioxidant effects [4]. Tannic acid has found industrial, pharmacological, and food applications. It is also used as an additive to medical products in burn treatment [5].

The term hydrogel is used to refer to the three-dimensional, cross-linked structures of hydrophilic polymers. Hydrogels are characterized by the ability to absorb large amounts of 
water or biological fluids. They balance the swelling process and form insoluble structures due to their three-dimensional network. Their affinity for water absorption is attributed to the presence of hydrophilic groups such as $-\mathrm{OH},-\mathrm{CONH}-,-\mathrm{CONH}_{2}-$, and $-\mathrm{SO}_{3} \mathrm{H}$ in the polymers forming hydrogel structures. The water sorption capacity of the hydrogel depends on several factors, such as the nature and density of the polymer used and the degree of cross-linking. Hydrogel structures share some physical properties that resemble those of living tissues more than any other class of synthetic materials. These features are attributed to their high water content, their softness and consistency, and the low interfacial tension of water or biological fluids. Hydrogels may be used as wound dressings to promote wound healing, and they ensure the moisture environment with gas permeability. Such materials may be also applied to fill the bone defects providing they allow for the cells' differentiation into osteoblasts.

Glyoxal is a chemical compound that contains two aldehyde groups. It is commonly used to cross-link polysaccharides [6-8]. It has cross-linking ability via acetal formation between the aldehyde groups of glyoxal and the hydroxyl groups of the glucosamine units of chitosan or through Schiff's base formation between the free amino groups of chitosan and the aldehyde groups of glyoxal [9]. Our previous studies showed that chitosan/tannic acid mixture additionally cross-linked by glyoxal and lyophilized leads to the obtainment of porous biocompatible structures [10].

In this study, hydrogels loaded with tannic acid were examined for potential medical applications as wound dressings or compresses for burns. The hydrogel structure, tannic acid release as well as mechanical properties and thermal stability of hydrogels were studied. Moreover, the tannic acid release profile was studied. The biological studies included the cell culture test on hDPC cells by their viability measurement. The proposed method of hydrogel preparation is fast and cheap and does not require any equipment compared to method published by us previously [10].

\section{Materials and Methods}

\subsection{Chitosan Characterization}

Chitosan (low molecular weight) has been purchased from the Sigma-Aldrich company (Poznan, Poland). The deacetylation degree (DD, \%) of chitosan and its viscosityaverage molecular weight were determined by the using viscometer (ViscoClock equipped with Ubbelohde ( SI Analytics, Mainz, Germany). Determined DD was 78\% [11], and the chitosan's molecular weight be equal to $1.8 \times 10^{6} \mathrm{D}$.

\subsection{Sample's Preparation}

Glyoxal (G), acetic acid, and tannic acid (TA), commercially available compounds, were purchased from the Sigma-Aldrich company (Poznan, Poland). Glyoxal solution (40 wt $\%$ in water; $\mathrm{M}_{\mathrm{w}}=58.04 \mathrm{~g} / \mathrm{mol}$ ) was used. The molecular weight of tannic acid was $1701.2 \mathrm{~g} / \mathrm{mol}$. The chitosan solution at $2 \%$ concentration was prepared in $0.1 \mathrm{M}$ acetic acid.

Chitosan-based hydrogels were prepared by glyoxal addition to chitosan solution. Different chitosan/glyoxal ratios were tested. It was noticed that a high concentration results in prolonged the exceeding gelation time. The addition of glyoxal was 5 and $10 \%$, where the most suitable glyoxal addition was $10 \%$. Thereby, the glyoxal was added drop by drop in amount $10 \%$ based on the weight of chitosan solution (room temperature). After each cross-linker addition, the mixture was mixed slowly on a magnetic stirrer for $1 \mathrm{~min}$ and left without mixing for $15 \mathrm{~min}$. As a result, semi-solid hydrogel in a gel-like form was obtained. Glyoxal is an aldehyde compound; it is important to not add more monomers that can react with chitosan, as glyoxal residues may be toxic. Thereby, hydrogels were then washed with distilled water to remove the unreacted cross-linker.

\subsection{Scanning Electron Microscope}

The morphology of the samples was studied using a scanning electron microscope (SEM) (LEO Electron Microscopy Ltd., Cambridge, UK). Hydrogels were placed into plastic 
plates and frozen $\left(-4{ }^{\circ} \mathrm{C}, 24 \mathrm{~h}\right)$. Then, samples were lyophilized (ALPHA 1-2 LDplus, CHRIST, $-20^{\circ} \mathrm{C}, 100 \mathrm{~Pa}, 24 \mathrm{~h}$ ). Such prepared dry hydrogels were covered by gold (thickness 5-10 nm) and studied. Scanning electron microscope images were made with magnification $500 \times$ and $10.0 \mathrm{kV}$.

\subsection{Thermal Properties}

Thermogravimetric analysis and differential thermal analysis (TG-DTA) were performed at a heating rate of $10^{\circ} \mathrm{C} / \mathrm{min}$ from 20 up to $750{ }^{\circ} \mathrm{C}$ in the nitrogen atmosphere by using TA Instruments SDT 2960 Simultaneous TGA-DTA (TA Instruments, Eschborn, Germany). From thermogravimetric curves, the characteristic temperature at a maximum decomposition rate of the investigated composites was determined for each type of hydrogel.

\subsection{Polyphenol Loading and Release}

The prepared chitosan-based hydrogels cross-linked with glyoxal were washed by water and then immersed in a polyphenol solution at different concentration values: 2, 5, 10 , and $20 \%$ (in water). The hydrogels were immersed for $24 \mathrm{~h}$ in $50 \mathrm{~mL}$ of TA solution (room temperature, sunlight exposure).

Hydrogels loaded with a polyphenol (tannic acid) were immersed in a phosphate buffered saline (PBS) solution ( $\mathrm{pH}=7.4$ ). The content of the polyphenolic compounds was determined using the Folin-Ciocalteu test [12]. The collected samples ( $1 \mathrm{~mL}$ of each one) were mixed with $0.5 \mathrm{~mL}$ Folin-Ciocalteu reagent. After $3 \mathrm{~min}, 1 \mathrm{~mL}$ of saturated $\mathrm{Na}_{2} \mathrm{CO}_{3}$ solution was added. The prepared mixtures were incubated at $40^{\circ} \mathrm{C}$ for $30 \mathrm{~min}$. The absorbance was measured at $725 \mathrm{~nm}$ using a UV-Vis spectrophotometer (UV-1800, Shimadzu, Kyoto, Japan). The experimental calibration curve was prepared for the standard solution at the concentration $0-2.5 \mathrm{mg} / \mathrm{mL}$ (gallic acid; $\mathrm{R}^{2}=09997$ ). The samples were collected 15, 30, 45, 60, 120, and $180 \mathrm{~min}$ after immersion.

Several mathematical equations may be used to define the dissolution profile. Once an appropriate model is selected, the drug release profile can be correlated with active compounds release kinetic models e.g., zero-order model, model based on first-order kinetic equation, Higuchi model, or Ritger-Peppas model [13].

\subsection{Mechanical Properties}

Mechanical properties were measured by the use of a mechanical testing machine (Shimadzu EZ-Test EZ-SX, Shimadzu, Kyoto, Japan). Hydrogels were placed in a 24hole plate to form cylindrical samples sized $20 \mathrm{~mm}$ in diameter and $13 \mathrm{~mm}$ in height for mechanical testing (Figure 1). The samples were introduced between two discs and compressed (the starting speed of $200 \mathrm{~mm} \mathrm{~min}^{-1}$; initial force of $0.1 \mathrm{~N}$ ). The compressive modulus (Young modulus for the compression process), the maximum tension, and the percentage deformation at maximum tension were determined with the Trapezium $X$ Texture program. The statistical analyses were made, and the results are shown as the average with the standard deviation.
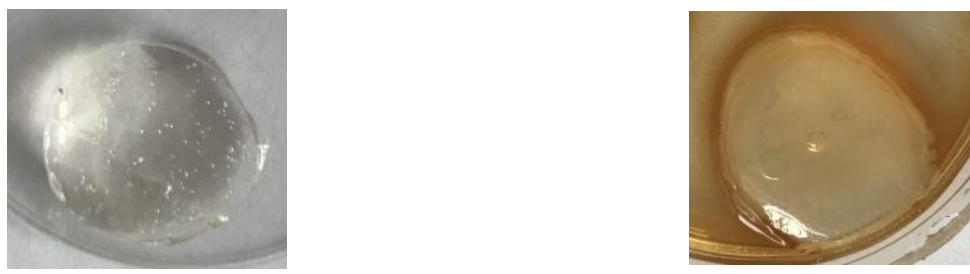

Figure 1. The shape of samples used for mechanical testing without immersion in TA (left) and after immersion in $20 \%$ TA solution (right). 


\subsection{Swelling Properties}

Hydrogels based on chitosan cross-linked by glyoxal were prepared. Swelling properties were studies for hydrogels without tannic acid immersion as well as after immersion in 2, 5, 10, and 20\% TA concentration for $24 \mathrm{~h}$. Then, hydrogels were placed in the PBS solution ( $\mathrm{pH}=7.4)$. The weight of hydrogels was studied every hour until $8 \mathrm{~h}$ of immersion. Then, swelling ratios were calculated using the Equation:

$$
\text { swelling }[\%]=\frac{m_{t}-m_{0}}{m_{0}} \times 100 \%
$$

where $m_{t}$ is the weight of the hydrogels after the time of immersion in PBS and $m_{0}$ is the weight before immersion.

\subsection{Blood Compatibility}

A sample of anticoagulated (by CPDA-1) sheep blood (with $0.2 \mathrm{~mL}$ ) was added to $10 \mathrm{~mL}$ of physiological saline solution containing different specimens at the same weight. Positive and negative samples were prepared by adding $0.2 \mathrm{~mL}$ of fresh blood to water and physiological saline, respectively. Hydrogels immersed without previous immersion in a polyphenol solution were used as control samples. All the test tubes were incubated at $37^{\circ} \mathrm{C}$. After $1 \mathrm{~h}$, the suspension was centrifuged at $1000 \mathrm{rpm}$ for $10 \mathrm{~min}$ [14-16]. The absorbance of supernatants was measured by a microplate reader, Multiscan FC (Thermo Fisher Scientific, Waltham, MA, USA), at $540 \mathrm{~nm}$. Each sample was prepared in triplicate. The hemolysis rate was calculated using the Equation:

$$
\text { rate of hemolysis }[\%]=\frac{[O D] \text { specimen }-[O D] \text { negative }}{[O D] \text { positive }-[O D] \text { negative }} \times 100 \%
$$

where $[O D]$ specimen is the absorbance for samples, $[O D]$ negative is absorbance for negative control (physiological saline), [OD]positive is absorbance for positive control (water). The experiment was carried out for hydrogels without washing, once washed by distilled water, without immersion in tannic acid solution, and after.

\subsection{Dental Culture Studies}

Dental pulp cells can be obtained from postnatal-, wisdom-, and/or deciduous teeth, providing a non-invasive alternative (compared to e.g., bone marrow) to obtain osteoprogenitor cells. Human dental pulp cells (hDPC) were obtained according to the method of Bakkar et al. [17] from the molar teeth of 31-43-year-old patients, both genders. Teeth were extracted due to orthodontic patients' treatments and were considered as surgical waste. Studies were carried out in agreement with the Local Bioethics Committee at the Jagiellonian University in Kraków; approval No. 1072.6120.253.2017 and informed consent was obtained from all donors.

Before cell culture, a sterilization technique (i.e., exposure to $70 \% \mathrm{EtOH}$ ) was assessed, and hydrogels cross-linked by tannic acid were examined for the potential leaking of tannic acid to the culture medium. Then, the hydrogels were sterilized in $70 \% \mathrm{EtOH}$, followed by $10 \mathrm{~min}$ exposure to UV light and then left overnight to dry. Following sterilization, the hydrogels were placed in separate wells of 24-well culture plates and seeded with $2 \times 10^{4}$ dental pulp cells (DPSC) suspended in $2 \mathrm{~mL}$ of culture medium. The latter was composed of alpha-minimum essential medium ( $\alpha \mathrm{MEM}$ ) supplemented with $10 \%$ fetal bovine serum (FBS) and antibiotics (penicillin/streptomycin 1\% mixture). The cells were assessed for viability after $24 \mathrm{~h}$ culture on hydrogels or on tissue culture plate (TCP). For cell viability assay (CellTiter96Aqueous One Solution Cell Proliferation Assay; Promega, Madison, WI, USA), hydrogels were washed gently and carefully with PBS, followed by the addition of $0.4 \mathrm{~mL} /$ well of $10 \%$ MTS reagent in phenol-free alpha-MEM. The plates were incubated at $37^{\circ} \mathrm{C}$ until the apparent change of color from yellow to brownish. Then, the colored media were transferred to individual wells in 96-well plates and the absorbance was recorded 
at $492 \mathrm{~nm}$ using a plate reader (SpectraMax iD3, Molecular Devices, San Jose, CA, USA). The results were expressed as a percentage of live cells on the studied hydrogels vs. live cells on control hydrogel (100\%) [18]. Data were analyzed by one-way analysis of variance (ANOVA) with Tukey's post hoc test and $p \leq 0.05$ was considered significant.

\section{Results}

\subsection{Scanning Electron Microscope}

Scanning electron microscope allows observing the structure of hydrogels (Figure 2). Hydrogel without tannic acid has a regular porous structure with open interconnected pores. Immersion in tannic acid solution results in the change in hydrogel's structure with closed pores.
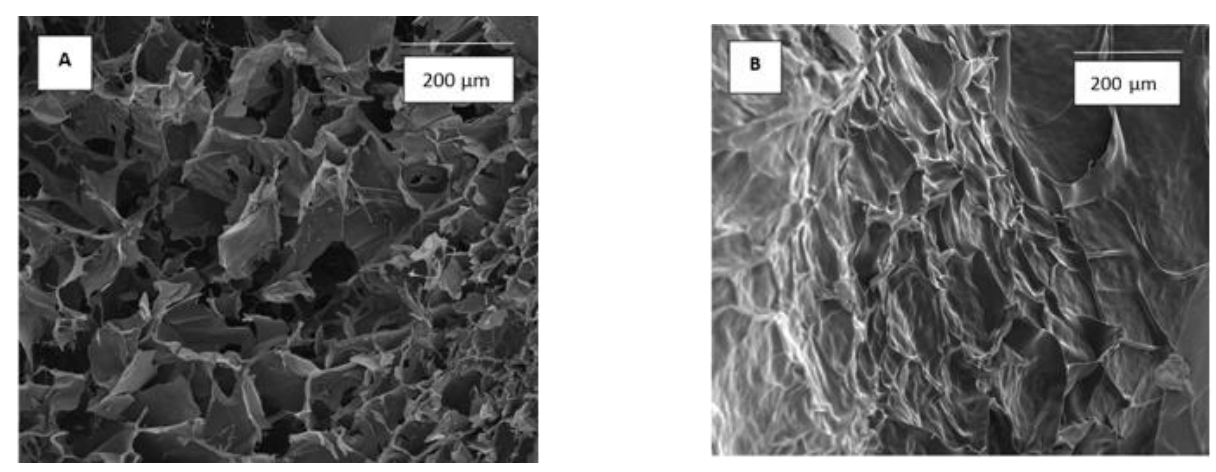

Figure 2. The structure of hydrogel without TA loading (A) and hydrogel loaded by immersion in tannic acid solution at $20 \%$ concentration (B); magnification $500 \times$.

\subsection{Thermal Properties}

The thermal behavior of hydrogels had been studied (Figure 3). The thermal properties of biopolymeric materials are interesting to be considered as biopolymers without crosslinking low denaturation temperature. Temperatures for maximum peaks were determined (Table 1). $\mathrm{T}_{\max }$ (3) could not be measured for hydrogel immersed in $2 \%$ tannic acid solution, as the peak was not well visible on the TG and DTA curves. Three steps of degradation of hydrogel were noticed. The highest weight loss was determined in the temperature range $20-170{ }^{\circ} \mathrm{C}$. It may be assigned to the elimination of water molecules present in hydrogel [19]. The shape of peak has been changed after the hydrogel immersion in tannic acid solution. As tannic acid has many hydroxyl groups, it interacts with water molecules, and their elimination is lower than from hydrogel without the presence of tannic acid. Two more regions in difference thermo gravimetric (DTG) curves may be distinguished. High peaks at $203.80^{\circ} \mathrm{C}$ observed for hydrogel without TA decreased rapidly with the presence of tannic acid. This peak may be assigned to the degradation of polymeric structure (chitosan). In addition, peaks present in higher temperature (above $268^{\circ} \mathrm{C}$ ) are assigned to the chitosan degradation. It may be observed for each type of hydrogel (without and with TA). However, the maximum temperature has been shifted to a higher temperature after the loading with TA. It is a result of hydrogen bonds present between chitosan and tannic acid, which stabilize the hydrogel structure.

Table 1. The results of DTA analysis with temperatures of maximum peaks

\begin{tabular}{cccc}
\hline Specimen & $\mathbf{T}_{\max }(\mathbf{1})\left[{ }^{\circ} \mathbf{C}\right]$ & $\mathbf{T}_{\max }(\mathbf{2})\left[{ }^{\circ} \mathbf{C}\right]$ & $\mathbf{T}_{\max }(\mathbf{3})\left[{ }^{\circ} \mathbf{C}\right]$ \\
\hline Without & 159.33 & 203.80 & 268.95 \\
2 & 107.21 & 200.52 & $-^{*}$ \\
5 & 83.53 & 109.21 & 277.20 \\
10 & 94.30 & 198.58 & 281.88 \\
20 & 117.02 & 195.90 & 278.84 \\
\hline
\end{tabular}

* The maximum temperature could not be measured. 
A

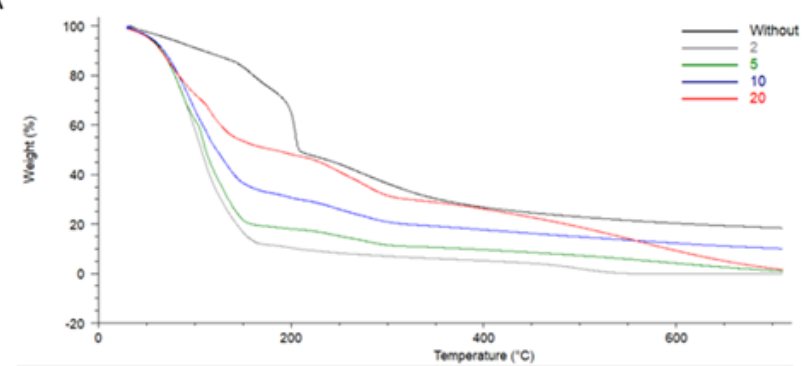

B

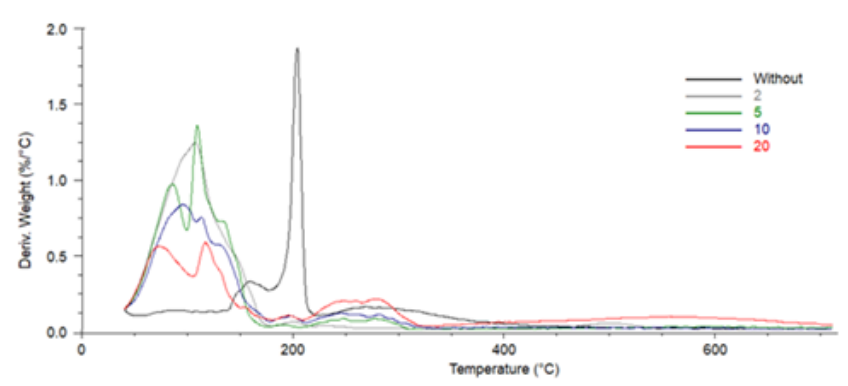

Figure 3. The TGA (A) and DTA (B) curves of chitosan-based hydrogels cross-linked by glyoxal without TA and after immersion in 2, 5, 10, and 20\% tannic acid solution.

\subsection{Polyphenol Loading and Release}

After polyphenol loading (Figure 4), the hydrogel changed color as a result of tannic acid loading. The released tannic acid concentration was determined by spectrophotometric analysis. The cumulative percentage concentration of released tannic acid in square root time dependence is shown in Figure 5. It may be observed that the dependence of the released concentration in time is similar for each solution. The highest released concentration was noticed for hydrogels immersed in $20 \%$ solution. Initially, the difference was small, but in the end of the experiment, the released concentration value for $20 \%$ solution was three times higher than for $2 \%$ solution. The polyphenol release studies showed that solutions with higher active substance concentrations used for hydrogels loaded with tannic acid are more effective for cosmetic and medical applications.

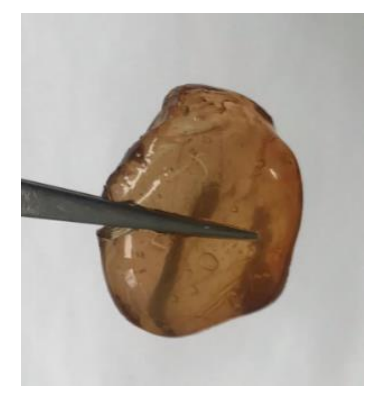

Figure 4. The hydrogel after immersion in $20 \%$ tannic acid solution.

Drug release data were fitted to the zero-order kinetic model and first-order kinetic model. In addition, the release data were fitted to the Higuchi and Ritger-Peppas equations to characterize the drug mechanism. The kinetic rate constants, release exponent $\mathrm{n}$ and values of $R^{2}$ for each model were calculated by linear least-squares regression analysis (Table 2) [20]. The most suitable model was selected based on the highest correlation coefficient values. The $\mathrm{R}^{2}$ of the Higuchi model is higher than for other models. This implies that the kinetic of tannic acid release from chitosan/glyoxal hydrogel pursues the Higuchi square root model (Figure 5). For the Ritger-Peppas model, the estimation of $n$ is utilized to describe the release mechanism. In our studies, the $n$ is in the range $0.43-0.85$. It suggests that the tannic acid release is controlled by both diffusion and relaxation of polymer chains [21]. 


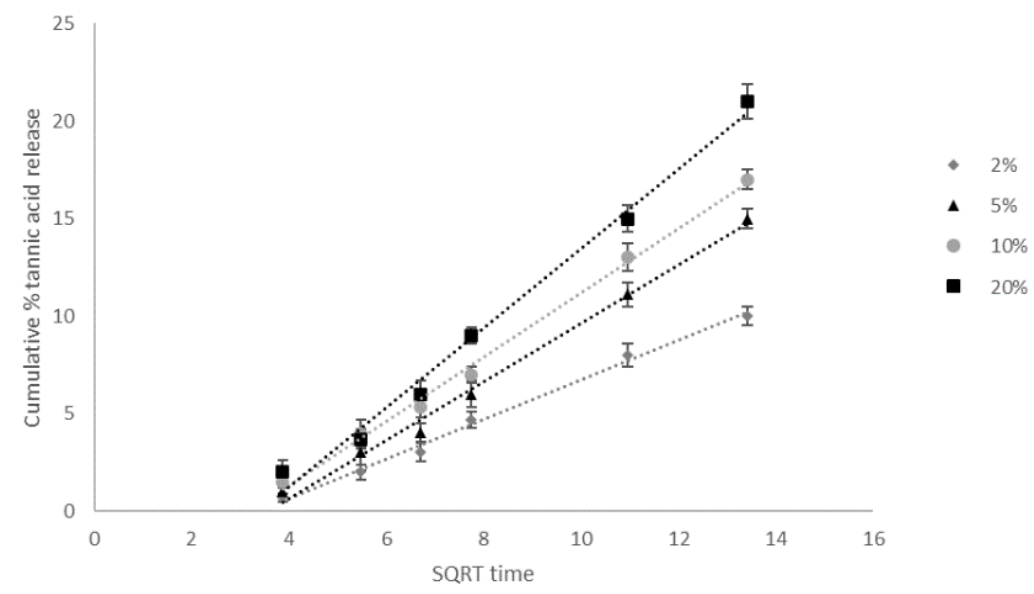

Figure 5. Tannic acid release from chitosan/glyoxal hydrogels curves of Higuchi square root model $(n=3)$.

Table 2. Kinetic parameters of tannic acid release from chitosan/glyoxal hydrogels.

\begin{tabular}{ccccccccccccc}
\hline \multirow{2}{*}{$\begin{array}{c}\text { Polyphenol } \\
\text { Solution [\%] }\end{array}$} & \multicolumn{2}{c}{ Zero-Order } & \multicolumn{2}{c}{ First-Order } & \multicolumn{2}{c}{ Higuchi } & \multicolumn{3}{c}{ Ritger-Peppas } \\
\cline { 2 - 10 } & $\mathbf{k}_{\mathbf{0}}$ & $\mathbf{R}^{\mathbf{2}}$ & $\mathbf{k}_{\mathbf{0}}$ & $\mathbf{R}^{\mathbf{2}}$ & $\mathbf{k}_{\mathbf{0}}$ & $\mathbf{R}^{\mathbf{2}}$ & $\mathbf{k}_{\mathbf{0}}$ & $\mathbf{R}^{\mathbf{2}}$ & $\mathbf{n}$ & 0.9611 & 0.6890 \\
\hline 2 & 0.0109 & 0.9213 & 0.0261 & 0.9331 & 0.1514 & 0.9942 & 0.6502 & 0.9625 & 0.7011 \\
\hline 5 & 0.0118 & 0.9256 & 0.0269 & 0.9381 & 0.1531 & 0.9934 & 0.6513 & 0.9625 \\
\hline 10 & 0.0122 & 0.9209 & 0.0275 & 0.9405 & 0.1533 & 0.9957 & 0.6530 & 0.9604 & 0.7288 \\
\hline 20 & 0.0125 & 0.9241 & 0.0281 & 0.9497 & 0.1542 & 0.9908 & 0.6538 & 0.9617 & 0.7301 \\
\hline
\end{tabular}

\subsection{Mechanical Properties}

The mechanical properties of hydrogels were determined by the compression test (Figure 6). The stress-strain curves for hydrogels are shown in Figure 7. The Young modulus of hydrogel without immersion in TA had slightly higher values than after immersion. However, all observed changes were not statistically significant. Thereby, it may be assumed that the immersion in TA solution did not change the hydrogel mechanical resistance.

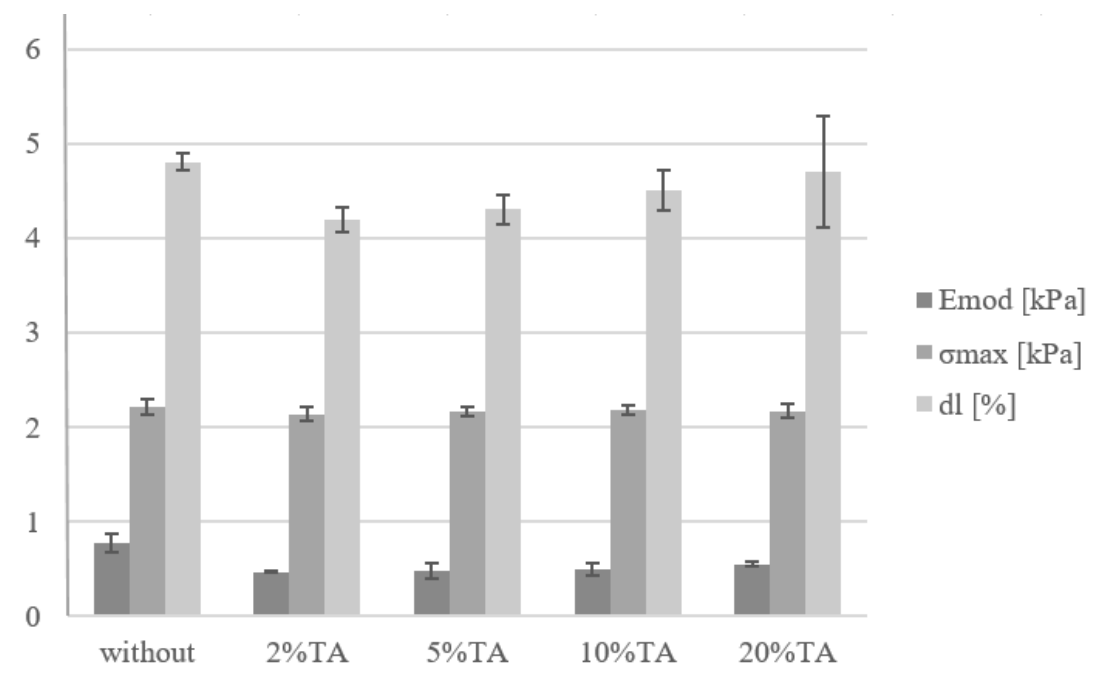

Figure 6. The mechanical parameters of hydrogel without immersion in tannic acid solution and of hydrogel immersed in TA solution at $2 \%, 5 \%, 10 \%$, and $20 \%$ concentration; for $p<0.05$, no statistically significant differences were observed (Emod—compressive modulus, omax-maxium tension, dl-percentage deformation at maximum tension). 


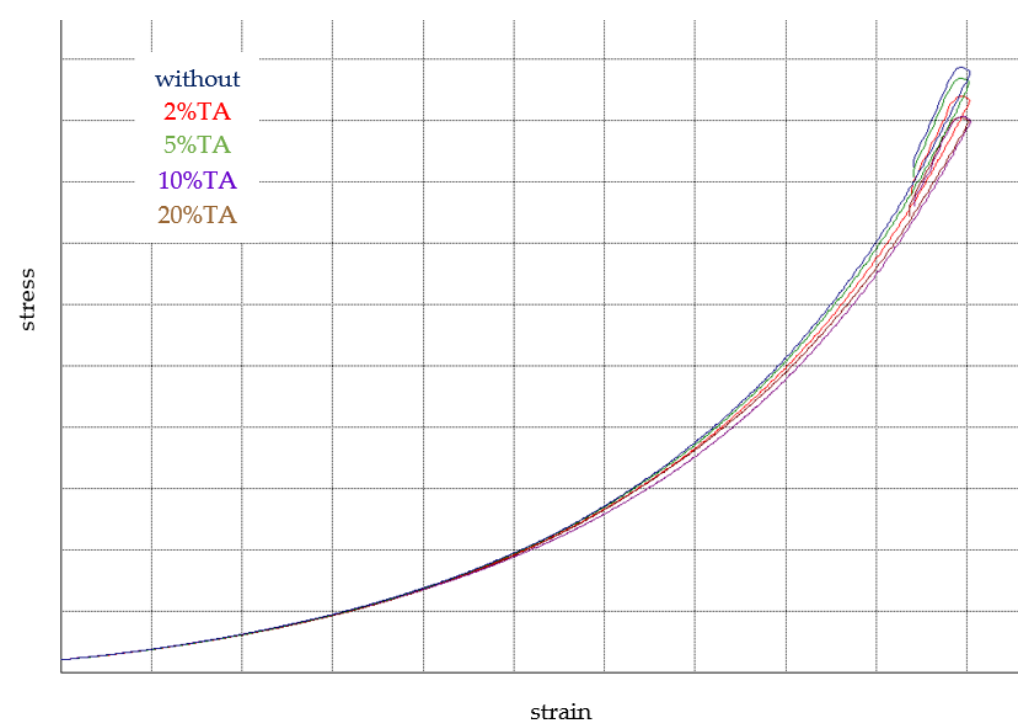

Figure 7. Stress-strain curves for hydrogel without immersion in tannic acid solution and of hydrogel immersed in TA solution at $2 \%, 5 \%, 10 \%$, and $20 \%$ concentration.

\subsection{Swelling Properties}

Hydrogels are materials with a high ability to swell. Hydrogels without immersion in TA solution showed high percentage swelling (Figure 8). Materials firstly immersed in TA solution had already swelled. Thereby, we did not observe a significant increase in the hydrogel weight. After $7 \mathrm{~h}$ of immersion in PBS, we observed a decrease in hydrogel weight, which may suggest that the dissolution process occurred.

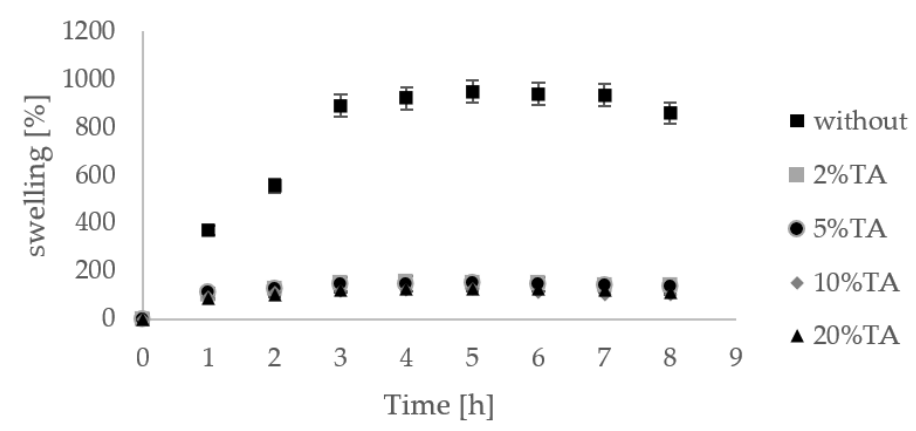

Figure 8. Swelling [\%] of hydrogels $(n=3)$ without immersion in TA and after immersion in TA solution at $2 \%, 5 \%, 10 \%$, and $20 \%$ concentration.

\subsection{Blood Compatibility}

The hemolysis rate is one of the most critical criteria when considering materials in blood-material contact for biomedical applications (Figure 9). Materials that show hemolysis lower than 5\% may be considered safe for biomedical applications [22]. The blood compatibility measurement results are listed in Table 3. Erythrocytes are sensitive to hemolysis due to shear stress [23]. The hemolysis rate for chitosan/glyoxal hydrogels without washing nor immersion in a polyphenol solution was $63.84 \%$, which means that such hydrogel is highly hemolytic. However, after washing with distilled water, the hemolysis rate was negative. The hydrogels washing by water results in the removing of unreacted glyoxal, and the hemolysis rate decreased. A similar observation was noticed for hydrogels immersed in tannic acid solution. As hydrogels were initially immersed in distilled water, a nonbonded cross-linking agent was removed. Then, loading by tannic acid did not increase the hemolysis rate. 


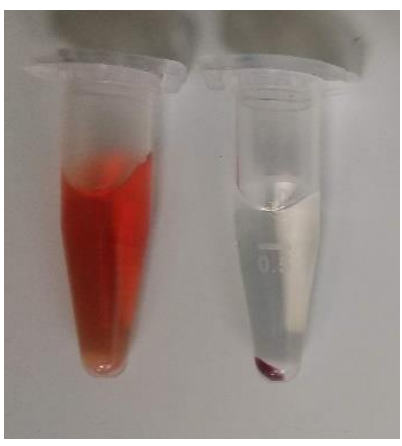

Figure 9. In vitro hemocompatibility assay left: hemolysis; right: no hemolysis.

Table 3. Hemolysis rate for chitosan/glyoxal hydrogels after immersion in polyphenol solutions at $2 \%, 5 \%, 10 \%$, and $20 \%$ concentration and without immersion for hydrogels washed and unwashed by distilled water.

\begin{tabular}{cc}
\hline Polyphenol Solution [\%] & Hemolysis Rate [\%] \\
\hline Without & $63.84 \pm 3.80$ \\
Without/washed & $-0.10 \pm 0.09$ \\
2 & $-0.09 \pm 0.07$ \\
5 & $-0.12 \pm 0.11$ \\
10 & $-0.16 \pm 0.08$ \\
20 & $-0.21 \pm 0.15$ \\
\hline
\end{tabular}

\subsection{Cell Culture Studies}

Our research has shown that the tested hydrogels immersed in TA solution do not show a cytotoxic effect on hDPC (Figure 10). A reduction in cell viability can be observed on hydrogels $2 \%$ and $20 \%$ in turn. The contents of $5 \%$ and $10 \%$ seem to stimulate the proliferation of cells grown on them. The hydrogel immersion in TA solution at concentrations of 2,5 , and $10 \%$ did not affect the increase of cytotoxicity of material.

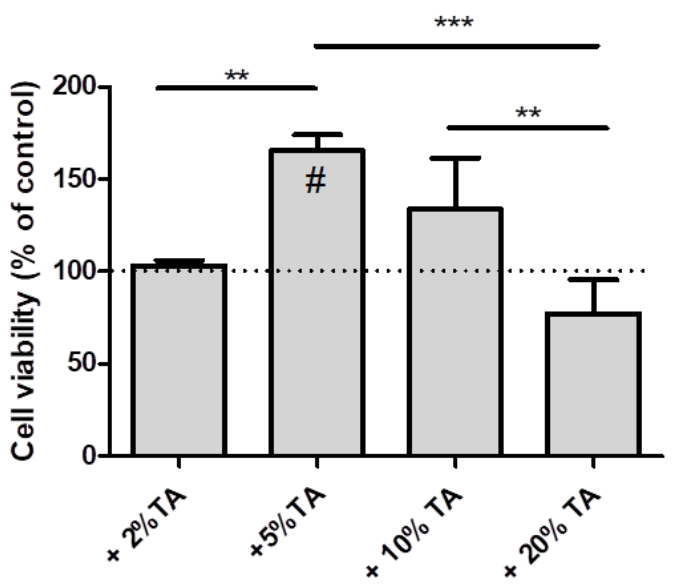

Figure 10. hPDL cells viability on tested hydrogels after $24 \mathrm{~h}$ of culture. Results are expressed as percentage change in cell viability compared to control hydrogel. \# indicates significant increased from control. Statistically significant differences $\left({ }^{* *} p \leq 0.05 ;{ }^{* * *} p \leq 0.001\right)$ within the groups.

\section{Discussion}

Hydrogels may be modified by the addition of different compounds to improve their physicochemical properties as well as biological response. Obtained hydrogels have a porous structure initially with open pores that after immersion in TA were closed. Chitosan-based hydrogels with gelatin showed better thermal stability than for pure chitosan. In addition, thermal properties were improved by $\mathrm{TiO}_{2}$ addition to the prepared 
hydrogels [24]. Tannic acid is able to form strong hydrogen interactions between polymer, both proteins and polysaccharides [25]. The two-step degradation of chitosan with TA hydrogels observed on TA and DTA curves may be assigned to the complex formation of chitosan-tannic acid (CTS-TA). The interactions between those two components have been considered in our previous work [26]. The complexation that occurs between chitosan and tannic acid result in the thermal stability improvement (Figure 11). The influence of chitosan complexation by alginate has been considered by Lv [27]. The authors strongly underline the importance of hydrogen bonds presence on the properties of the hydrogels as final products that are obtained.

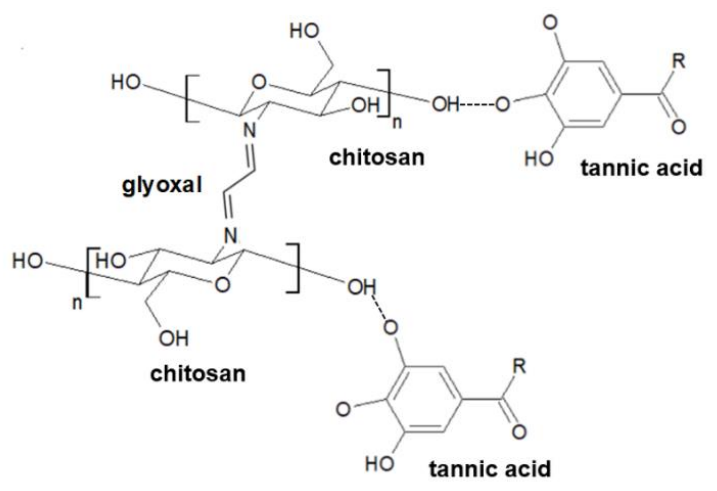

Figure 11. The schematic cross-linking mechanism of chitosan by glyoxal.

The main application of hydrogels is as drug delivery devices [28]. We confirmed that a simple method of hydrogels immersion in tannic acid is effective for TA incorporation into a hydrogel. The immersion in TA did not affect the changes in the mechanical properties of hydrogels. Since chitosan-based hydrogels may be effectively soaked by tannic acid, they may deliver such active compounds as TA. Tannic acid may act as an anti-inflammatory factor, and it has antimicrobial and anticancer properties [29-31]. Chitosan-based materials have been considered as effective drug delivery systems of phenolic acids [32-34]. As a result, such materials enriched by polyphenolic acids may be used as food packaging or dressing materials as well.

As it was discussed by Liu [35], the introduction of phenolic units into the chitosan backbone is a great alternative to improve its physicochemical properties, but also, it remarkably increases material biological features. We reported that chitosan/tannic acid films show a selective influence on the cells that depends on their type-human bone marrow stromal cells (BMSC), human melanoma (MNT-1), human osteosarcoma (SaOS), and human keratinocytes (HaCaT) cells [36]. In the present study, we proposed chitosanbased hydrogels cross-linked by glyoxal. Glyoxal is an aldehyde that effectively cross-links amine groups present in the chitosan polymeric chain. Given that monomers of aldehydes are toxic, there is a need to determine the lowest amount of glyoxal to bind cross-linker completely to polymer [37]. It is important as unreacted cross-linker monomers may be toxic for the human body and should not remain in the final products and nonbonded.

There is information in the literature about the positive effect of TA-enriched scaffolds on osteogenic differentiation in BMSC cells [38]. Our research shows a stimulating effect on the proliferation of hPDL cells, which are also considered osteogenic [39,40]. However, checking the osteogenic properties of the studied hydrogels requires deeper biological analyses, which was not currently our goal in this article. Glyoxal was also studied by us before as a safe cross-linker useful to obtain stable scaffolds based on chitosan and tannic acid mixture [10]. However, it is necessary to remove underacted glyoxal as it may have a cytotoxic effect on cells.

\section{Conclusions}

Chitosan-based hydrogels were obtained successfully by the addition of glyoxal as a cross-linker. In addition, we have shown that polyphenols may be loaded to such hydrogels 
by simple hydrogel immersion in polyphenol solutions. Our studies suggest that obtained hydrogels may be used as phenolic acid delivery systems. All hydrogels were nonhemolytic and therefore they are safe to be applied in contact with blood. In addition, hydrogels did not show the cytotoxic effect. We believe the results of this work suggest that the obtained hydrogels may prove useful for several biomedical applications, such as wound dressings or bone-related treatment procedures.

Author Contributions: Conceptualization, B.K.-S.; methodology, B.K.-S., M.M.-S., O.M., K.t., A.M.O.; formal analysis, O.M., M.M.-S., K.E.; investigation, B.K.-S.; resources, B.K.-S., A.M.O.; writing—original draft preparation, B.K.-S.; writing—review and editing, M.M.-S., A.M.O.; visualization, B.K.-S.; supervision, B.K.-S.; funding acquisition, B.K.-S., A.M.O. All authors have read and agreed to the published version of the manuscript.

Funding: This research was funded by National Science Center, grant number UMO-2016/21/B/NZ5/ 00217 (A.M.O.) and Nicolaus Copernicus University in Torun, grant number 282/2021 IDUB (B.K.-S.).

Institutional Review Board Statement: The study was conducted according to the guidelines of the Declaration of Helsinki, and approved by the Local Bioethics Committee at the Jagiello-nian University in Kraków (protocol code No. 1072.6120.253.2017).

Informed Consent Statement: Informed consent was obtained from all subjects involved in the study.

Data Availability Statement: The data presented in this study are available on request from the corresponding author. The data are not publicly available due to 01.2023 .

Conflicts of Interest: The authors state that there are no conflicts of interest.

\section{References}

1. Smith, R.; Russo, J.; Fiegel, J.; Brogden, N. Antibiotic Delivery Strategies to Treat Skin Infections When Innate Antimicrobial Defense Fails. Antibiotics 2020, 9, 56. [CrossRef]

2. El-Banna, F.S.; Mahfouz, M.E.; Leporatti, S.; El-Kemary, M.; Hanafy, N.A.N. Chitosan as a Natural Copolymer with Unique Properties for the Development of Hydrogels. Appl. Sci. 2019, 9, 2193. [CrossRef]

3. Naskar, S.; Sharma, S.; Kuotsu, K. Chitosan-based nanoparticles: An overview of biomedical applications and its preparation. J. Drug Deliv. Sci. Technol. 2019, 49, 66-81. [CrossRef]

4. Lopes, G.K.; Schulman, H.M.; Hermes-Lima, M. Polyphenol tannic acid inhibits hydroxyl radical formation from Fenton reaction by complexing ferrous ions ${ }^{1}$. Biochim. Biophys. Acta (BBA) Gen. Subj. 1999, 1472, 142-152. [CrossRef]

5. Khan, N.S.; Ahmad, A.; Hadi, S. Anti-oxidant, pro-oxidant properties of tannic acid and its binding to DNA. Chem. Interact. 2000, 125, 177-189. [CrossRef]

6. Wang, L.; Stegemann, J.P. Glyoxal crosslinking of cell-seeded chitosan/collagen hydrogels for bone regeneration. Acta Biomater. 2011, 7, 2410-2417. [CrossRef] [PubMed]

7. Jawad, A.H.; Norrahma, S.S.A.; Hameed, B.; Ismail, K. Chitosan-glyoxal film as a superior adsorbent for two structurally different reactive and acid dyes: Adsorption and mechanism study. Int. J. Biol. Macromol. 2019, 135, 569-581. [CrossRef]

8. Gupta, K.; Jabrail, F.H. Glutaraldehyde and glyoxal cross-linked chitosan microspheres for controlled delivery of centchroman. Carbohydr. Res. 2006, 341, 744-756. [CrossRef] [PubMed]

9. Monier, M.; Ayad, D.; Wei, Y.; Sarhan, A. Preparation of cross-linked chitosan/glyoxal molecularly imprinted resin for efficient chiral resolution of aspartic acid isomers. Biochem. Eng. J. 2010, 51, 140-146. [CrossRef]

10. Kaczmarek-Szczepańska, B.; Miłek, O.; Michalska-Sionkowska, M.; Osyczka, A.M. Biostudies of scaffolds based on chitosan/tannic acid cross-linked by glyoxal. Mater. Lett. 2021, 292, 129667. [CrossRef]

11. de Moura, C.M.; de Moura, J.M.; Madeira Soares, N.; de Almeida Pinto, L.A. Evaluation of molar weight and deacetylation degree of chitosan during chitin deacetylation reaction: Used to produce biofilm. Chem. Eng. Process 2011, 50, 351-355. [CrossRef]

12. Kozlowska, J.; Prus, W.; Stachowiak, N. Microparticles based on natural and synthetic polymers for cosmetic applications. Int. J. Biol. Macromol. 2019, 129, 952-956. [CrossRef]

13. Agili, F.A.; Aly, S.F.M. Physicochemical characterization and release properties of oral drug delivery: A pH-sensitive nanocomposite based on sodium alginate-pectin-tannic acid-silver. Polym. Polym. Comp. 2019, 28, 598-608. [CrossRef]

14. Madhyastha, H.; Madhyastha, R.; Thakur, A.; Kentaro, S.; Dev, A.; Singh, S.; Chandrashekharappa, B.R.; Kumar, H.; Acevedo, O.; Nakajima, Y.; et al. c-Phycocyanin primed silver nano conjugates: Studies on red blood cell stress resilience mechanism. Colloids Surf. B Biointerfaces 2020, 194, 111211. [CrossRef] [PubMed]

15. Lyu, Y.; Ren, H.; Yu, M.; Li, X.; Li, D.; Mu, C. Using oxidized amylose as carrier of linalool for the development of antibacterial wound dressing. Carbohydr. Polym. 2017, 174, 1095-1105. [CrossRef] [PubMed]

16. Zhou, H.Y.; Zhang, Y.P.; Zhang, W.F.; Chen, X.G. Biocompatibility and characteristics of injectable chitosan-based thermo-sensitive hydrogel for drug delivery. Carbohyd. Polym. 2011, 83, 1643-1651. [CrossRef] 
17. Bakkar, M.; Liu, Y.; Fang, D.; Stegen, C.; Su, X.; Ramamoorthi, M.; Lin, L.-C.; Kawasaki, T.; Makhoul, N.; Pham, H.; et al. A Simplified and Systematic Method to Isolate, Culture, and Characterize Multiple Types of Human Dental Stem Cells from a Single Tooth. Breast Cancer 2017, 1553, 191-207. [CrossRef]

18. Łukowicz, K.; Zagrajczuk, B.; Nowak, A.; Niedźwiedzki, Ł.; Laczka, M.; Cholewa-Kowalska, K.; Osyczka, A.M. The role of $\mathrm{CaO} / \mathrm{SiO}_{2}$ ratio and $\mathrm{P}_{2} \mathrm{O}_{5}$ content in gel-derived bioactive glass-polymer composites in the modulation of their bioactivity and osteoinductivity in human BMSCs. Mater. Sci. Eng. C 2020, 109, 110535. [CrossRef]

19. Alemdar, N. Synthesis of chitosan-based hydrogel by using photopolymerization technique. Anadolu Univ. J. Sci. Technol. Appl. Sci. Eng. 2016, 17, 391-400. [CrossRef]

20. Gierszewska, M.; Ostrowska-Czubenko, J.; Chrzanowska, E. Characteristics of ascorbic acid release from tpp-crosslinked chitosan/alginate polyelectrolyte complex membranes. Prog. Chem. Appl. Chitin Deriv. 2018, XXIII, 76-87. [CrossRef]

21. Hu, X.; Yan, L.; Wang, Y.; Xu, M. Microwave-assisted synthesis of nutgall tannic acid-based salecan polysaccharide hydrogel for tunable release of $\beta$-lactoglobulin. Int. J. Biol. Macromol. 2020, 161, 1431-1439. [CrossRef] [PubMed]

22. Weber, M.; Steinle, H.; Golombek, S.; Hann, L.; Schlensak, C.; Wendel, H.P.; Avci-Adali, M. Blood-contacting Biomaterials: In vitro evaluation of the hemocompatibility. Front. Bioeng. Biotechnol. 2018, 6, 99. [CrossRef]

23. Pires, A.L.R.; Motta, L.A.; Dias, A.M.A.; de Sousa, H.C.; Moraes, A.M.; Braga, M.E.M. Towards wound dressings with im-proved properties: Effects of poly(dimethylsiloxane) on chitosan-alginate films loaded with thymol and beta-carotene. Mater. Sci. Eng. C 2018, 93, 595-605. [CrossRef] [PubMed]

24. Guo, N.; Zhang, L.; Wang, J.; Wang, S.; Zou, Y.; Wang, X. Novel fabrication of morphology tailored nanostructures with Gelatin/Chitosan Co-polymeric bio-composited hydrogel system to accelerate bone fracture healing and hard tissue nursing care management. Process. Biochem. 2020, 90, 177-183. [CrossRef]

25. Kaczmarek, B.; Mazur, O. Collagen-Based Materials Modified by Phenolic Acids-A Review. Materials 2020, 13, 3641. [CrossRef] [PubMed]

26. Kaczmarek, B.; Wekwejt, M.; Nadolna, K.; Owczarek, A.; Mazur, O.; Pałubicka, A. The mechanical properties and bactericidal degradation effectiveness of tannic acid-based thin films for wound care. J. Mech. Behav. Biomed. Mater. 2020, 110, 103916. [CrossRef]

27. Lv, X.; Zhang, W.; Liu, Y.; Zhao, Y.; Zhang, J.; Hou, M. Hygroscopicity modulation of hydrogels based on carboxymethyl chitosan/Alginate polyelectrolyte complexes and its application as pH-sensitive delivery system. Carbohyd. Polym. 2018, 198, 86-93. [CrossRef]

28. Onaciu, A.; Munteanu, R.A.; Moldovan, C.S.; Berindan-Neagoe, I. Hydrogels Based Drug Delivery Synthesis, Characterization and Administration. Pharmaceutics 2019, 11, 432. [CrossRef]

29. Kaczmarek, B. Tannic Acid with Antiviral and Antibacterial Activity as A Promising Component of Biomaterials-A Minireview. Materials 2020, 13, 3224. [CrossRef]

30. Shahidi, F.; Yeo, J. Bioactivities of Phenolics by Focusing on Suppression of Chronic Diseases: A Review. Int. J. Mol. Sci. 2018, 19, 1573. [CrossRef]

31. Abu Zarin, M.; Wan, H.Y.; Isha, A.; Armania, N. Antioxidant, antimicrobial and cytotoxic potential of condensed tannins from Leucaena leucocephala hybrid-Rendang. Food Sci. Hum. Wellness 2016, 5, 65-75. [CrossRef]

32. Talón, E.; Trifkovic, K.T.; Vargas, M.; Chiralt, A.; González-Martínez, C. Release of polyphenols from starch-chitosan based films containing thyme extract. Carbohydr. Polym. 2017, 175, 122-130. [CrossRef] [PubMed]

33. Dadwal, V.; Bhatt, S.; Joshi, R.; Gupta, M. Development and characterization of controlled released polyphenol rich microencapsulate of Murraya koenigii bark extract. J. Food Process. Preserv. 2020, 44, 14438. [CrossRef]

34. Lišková, J.; Douglas, T.E.; Beranová, J.; Skwarczyńska, A.; Božič, M.; Samal, S.K.; Modrzejewska, Z.; Gorgieva, S.; Kokol, V.; Bačáková, L. Chitosan hydrogels enriched with polyphenols: Antibacterial activity, cell adhesion and growth and mineralization. Carbohydr. Polym. 2015, 129, 135-142. [CrossRef] [PubMed]

35. Liu, J.; Pu, H.; Liu, S.; Kan, J.; Jin, C. Synthesis, characterization, bioactivity and potential application of phenolic acid grafted chitosan: A review. Carbohydr. Polym. 2017, 174, 999-1017. [CrossRef]

36. Kaczmarek, B.; Miłek, O.; Nadolna, K.; Owczarek, A.; Kleszczyński, K.; Osyczka, A.M. Normal and cancer cells response on the thin films based on chitosan and tannic acid. Toxicol. Vitr. 2020, 62, 104688. [CrossRef]

37. Hasan, M.M.; Khan, M.N.; Haque, P.; Rahman, M.M. Novel alginate-di-aldehyde cross-linked gelatin/nano-hydroxyapatite bioscaffolds for soft tissue regeneration. Int. J. Biol. Macromol. 2018, 117, 1110-1117. [CrossRef]

38. Zhang, W.; Ling, C.; Liu, H.; Zhang, A.; Mao, L.; Wang, J.; Chao, J.; Backman, L.J.; Yao, Q.; Chen, J. Tannic acid-mediated dual peptide-functionalized scaffolds to direct stem cell behavior and osteochondral regeneration. Chem. Eng. J. 2020, $396,125232$. [CrossRef]

39. Liu, J.; Zhao, Z.; Ruan, J.; Weir, M.D.; Ma, T.; Ren, K.; Schneider, A.; Oates, T.W.; Li, A.; Zhao, L.; et al. Stem cells in the periodontal ligament differentiated into osteogenic, fibrogenic and cementogenic lineages for the regeneration of the periodontal complex. $J$. Dent. 2020, 92, 103259. [CrossRef] [PubMed]

40. Wescott, D.; Pinkerton, M.; Gaffey, B.; Beggs, K.; Milne, T.; Meikle, M. Osteogenic Gene Expression by Human Periodontal Ligament Cells under Cyclic Tension. J. Dent. Res. 2007, 86, 1212-1216. [CrossRef] 